\title{
A Regularized Inverse Approach to Ultrasonic Pulse-Echo Imaging
}

\author{
Roberto Lavarello*, Student Member, IEEE, Farzad Kamalabadi, Member, IEEE, and \\ William D. O'Brien, Jr., Fellow, IEEE
}

\begin{abstract}
An inverse-theoretic approach to ultrasonic pulse-echo imaging based on nonquadratic regularization is presented, and its effectiveness is investigated computationally by: 1) evaluating the quality of the reconstruction of speckle-based images as a function of the transmit-receive bandwidth and focal number of the transducer; 2) comparing the reconstructed images with those obtained by using conventional B-mode imaging. The L-curve and the generalized cross-validation methods were evaluated as automatic regularization parameter selection techniques. The inversion using regularization produced better results than conventional B-mode imaging for high signal-to-noise ratios (SNRs). A lower bound of $30 \mathrm{~dB}$ for the SNR was found for this study, below which several of the image features were lost during the reconstruction process in order to control the distortion due to the noise.
\end{abstract}

Index Terms-Inverse problems, pulse-echo imaging, regularization, ultrasound imaging.

\section{INTRODUCTION}

B -MODE, array imaging, and synthetic aperture focusing techniques (SAFT) have been studied in acoustic imaging for a long time and their limitations are well documented [1]-[3]. Such techniques suffer from spatial resolution limitations due to the finite size of the resolution cell of the imaging system, i.e., the area (or volume) that corresponds to the smallest resolvable detail, and contrast resolution limitations due to the presence of sidelobes. Because ultrasonic imaging is coherent, when there are many scatterers randomly distributed in a resolution cell, their reflections interfere constructively or destructively depending on their positions relative to the transducer, thus generating spots (speckle) of brightness and darkness in the image. The resulting image has a granular appearance resulting in speckle-based images [4]. Speckle is very common in ultrasound medical imaging because tissue is a semitransparent media. If the point spread function of the imaging system has a wide mainlobe or high-amplitude sidelobes, the spots generated by the speckle can mask regions of low contrast. Inverse problem approaches [5] and regularization techniques, such as truncated singular value decomposition (TSVD) [6], Tikhonov [7], total variation [8], total least squares [9], and others [10]-[14], have been proposed for acoustic

Manuscript received October 5, 2005; revised February 22, 2006. Asterisk indicates corresponding author.

*R. Lavarello is with the Department of Electrical and Computer Engineering, University of Illinois at Urbana-Champaign, Urbana, IL 61801 USA (e-mail: lavarell@uiuc.edu).

F. Kamalabadi and W. D. O'Brien, Jr. are with the Department of Electrical and Computer Engineering, University of Illinois at Urbana-Champaign, Urbana, IL 61801 USA.

Digital Object Identifier 10.1109/TMI.2006.873297 imaging applications and their effectiveness has been explored to a limited extent. It is of special importance to compare the performance of this class of techniques with that which can be obtained using conventional acoustic imaging methods. In this paper, speckle-based images are proposed as targets of reconstruction because of the limitations of conventional techniques in dealing with this type of images. The goal of this paper is to explore the feasibility and effectiveness of a generalized Tikhonov regularization technique by 1) evaluating the quality of the reconstruction of speckle-based images as a function of imaging parameters (i.e., $-6 \mathrm{~dB}$ transmit-receive bandwidth $(B W)$ and focal number $\left(f^{\#}\right)$ of the transducer) and 2) comparing the reconstructed images with those obtained by using conventional B-mode imaging.

\section{Methodology}

\section{A. Continuous Forward Model}

The key concept for acoustic imaging is that an acoustic wave propagating through a medium is scattered when inhomogeneities are encountered. Thus, inhomogeneities inside a volume can be detected by radiating it with an acoustic wave and measuring the scattered energy with sensors positioned at different locations. The goal of ultrasonic pulse-echo imaging is to provide a graphical representation of the acoustic reflectivity function, that is, the distribution of the changes in acoustic impedance that cause the scattering of the incident wave. For monostatic pulse-echo ultrasonic imaging, a single-element transducer is placed at scanning positions $\overrightarrow{\rho_{0}}$ along a trajectory (typically a straight line); in each scanning position, the transducer is excited to propagate an acoustic pulse into the region of interest (ROI), and is also used to receive the corresponding backpropagated acoustic echo signals. The time signal received by the transducer when only one point scatterer is present in the ROI is known as the spatially variant impulse response of the transducer, which depends on the relative position between the transducer and the scatterer, $\overrightarrow{\rho_{r}}$, and is denoted by $s\left(\overrightarrow{\rho_{r}}, t\right)$. The pulse-echo data received when the transducer is located at $\overrightarrow{\rho_{0}}$ are denoted by $g\left(\overrightarrow{\rho_{0}}, t\right)$. The reflectivity function at the point $\vec{\rho}$ is denoted by $r(\vec{\rho})$. If multiple scattering can be neglected, the contribution of each point $\vec{\rho} \in$ ROI to the signal $g\left(\overrightarrow{\rho_{0}}, t\right)$ will be given by the spatially variant impulse response $s\left(\vec{\rho}-\overrightarrow{\rho_{0}}, t\right)$ weighted by the corresponding value of the reflectivity function $r(\vec{\rho})$. For a two-dimensional (2-D) ROI, the signal $g\left(\overrightarrow{\rho_{0}}, t\right)$ can be expressed in integral form as

$$
g\left(\overrightarrow{\rho_{0}}, t\right)=\iint_{R O I} s\left(\vec{\rho}-\overrightarrow{\rho_{0}}, t\right) r(\vec{\rho}) d \vec{\rho} .
$$




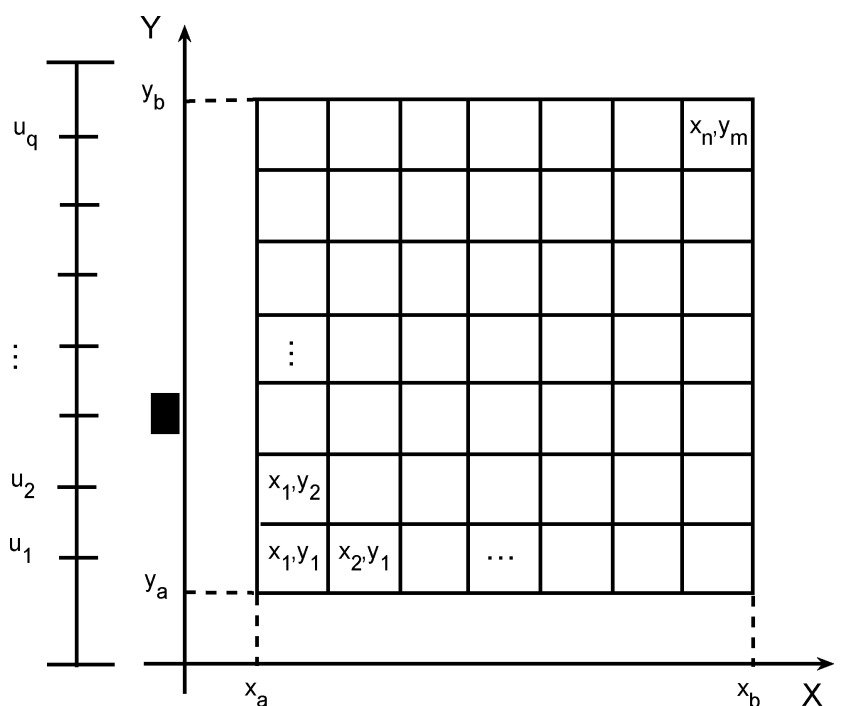

Fig. 1. Configuration for the monostatic pulse-echo imaging inversion. The $\mathrm{ROI}$ is a rectangular region with $x \in\left[x_{a}, x_{b}\right]$ and $y \in\left[y_{a}, y_{b}\right]$, discretized in $n$ by $m$ pixels. The transducer is shown as a black rectangle, and it scans the ROI along the $y$ axis in sampling positions $(0, u)$.

\section{B. Discrete Forward Model}

The continuous model must be discretized in order to solve for the reflectivity distribution. The ROI has been modeled as a rectangular region with axial (or beam) axis $x$ and lateral (or scanning) axis $y$, as shown in Fig. 1. The transducer is moved along the $y$ axis and the pulse-echo data are acquired at locations $\left(0, u_{l}\right)$. The received signals are sampled at discrete values of time $t$ denoted by $t_{k}$. The sample at time $t_{k}$ of the signal recorded at the position $\left(0, u_{l}\right)$ is denoted by $g_{u_{l}}\left(t_{k}\right)$. The ROI is also discretized, with the discrete values of $x$ and $y$ denoted by $x_{i}$ and $y_{j}$, respectively. The reflectivity of a point at the position $\left(x_{i}, y_{j}\right)$ inside the ROI is denoted by $r_{x_{i}, y_{j}}$. The spatially variant impulse response of the transducer, when a point target of unitary amplitude is located at the coordinates $(\hat{x}, \hat{y})$ relative to the position of the transducer, is denoted by $s_{\hat{x}, \hat{y}}(t)$.
The 2-D sequences $r_{x_{i}, y_{j}}$ and $g_{u_{l}}(t)$ are stacked as vectors to form a pair of one-dimensional sequences, which allows the problem to be represented as a set of linear equations [15]. With this reordering, the forward model can be written as the linear system $\mathbf{S r}=\mathbf{g}$ depicted in (2) at the bottom of the page, where the matrix $\mathbf{S}$ and the vectors $\mathbf{r}$ and $\mathbf{g}$ represent the linear forward model, the unknown variables (in this case, the reflectivity distribution) and the measurements (the pulse-echo data), respectively. (See equation at bottom of page)

\section{Regularized Inversion of the Discrete Forward Model}

The minimum mean square error (MMSE) solution to a linear system such as the one described by (2) is calculated by finding the solution to

$$
\hat{\mathbf{r}}=\underset{\mathbf{r}}{\arg \min }\left\{\|\mathbf{g}-\mathbf{S r}\|_{2}^{2}\right\} .
$$

The solution to (3) can be found using the pseudoinverse of the forward model matrix $\mathbf{S}$ [16]. The singular value decomposition (SVD) of $\mathbf{S}$ can be expressed as $\mathbf{S}=\mathbf{U D V}^{\mathbf{T}}$, where $\mathbf{U}$ and $\mathbf{V}$ are unitary matrices and $\mathbf{D}$ is a diagonal matrix with the singular values of $\mathbf{S}$ in decreasing order over the main diagonal. The pseudoinverse $\mathbf{S}^{+}$is then defined as $\mathbf{S}^{+}=\mathbf{V} \mathbf{D}^{+} \mathbf{U}^{\mathbf{T}}$, where $\mathbf{D}^{+}$is a diagonal matrix constructed by transposing $\mathbf{D}$ and replacing the nonzero entries by their reciprocals. The solution to the inverse problem in (2) is given by

$$
\hat{\mathbf{r}}=\mathbf{S}^{+} \mathbf{g}=\sum_{i=1}^{n} \frac{\mathbf{u}_{\mathbf{i}}^{\mathbf{T}} \mathbf{g}}{\sigma_{i}} \mathbf{v}_{\mathbf{i}}
$$

where $\sigma_{i}$ is the $i$ th singular value of the forward model matrix $\mathbf{S}, \mathbf{u}_{\mathbf{i}}$ and $\mathbf{v}_{\mathbf{i}}$ are the $i$ th columns of the unitary matrices $\mathbf{U}$ and $\mathbf{V}$, respectively, and $n$ is the number of singular values of $\mathbf{S}$. In practice, there will be noise present in the measurements, and (4) can be rewritten as

$$
\hat{\mathbf{r}}=\sum_{i=1}^{n} \frac{\mathbf{u}_{\mathbf{i}}^{\mathbf{T}} \mathbf{y}}{\sigma_{i}} \mathbf{v}_{\mathbf{i}}+\sum_{i=1}^{r} \frac{\mathbf{u}_{\mathbf{i}}^{\mathbf{T}} \mathbf{w}}{\sigma_{i}} \mathbf{v}_{\mathbf{i}}
$$

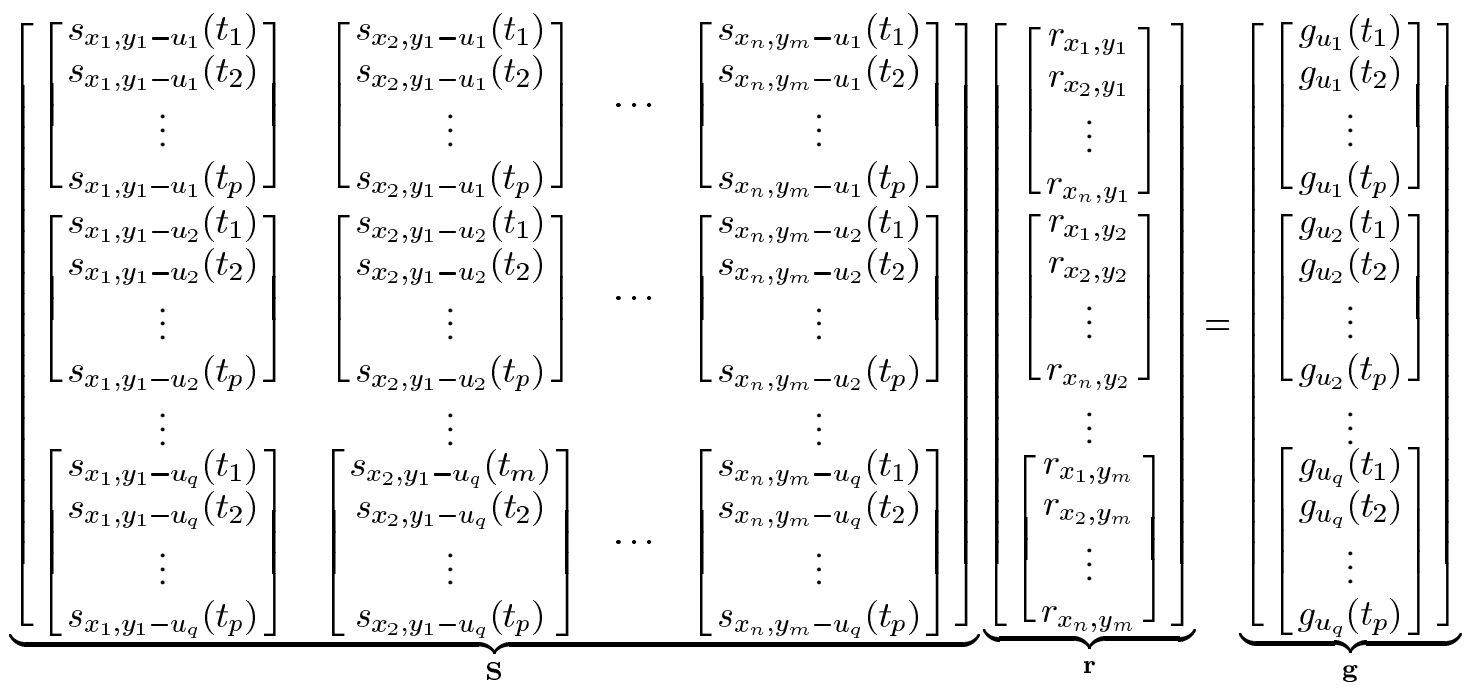


where $\mathbf{y}$ is the noiseless data as predicted by the forward model and $\mathbf{w}$ is the additive noise present in the measurements (i.e., $\mathrm{g}=\mathbf{y}+\mathbf{w})$. Even though the singular values $\sigma_{i}$ decrease for increasing $i$, the terms $\mathbf{u}_{\mathbf{i}}^{\mathbf{T}} \mathbf{y}$ decrease accordingly and the contributions of $\mathbf{y}$ are bounded. However, the terms $\mathbf{u}_{\mathbf{i}}^{\mathbf{T}} \mathbf{w}$ do not satisfy the discrete Picard condition (i.e., on average, the terms $\mathbf{u}_{\mathbf{i}}^{\mathbf{T}} \mathbf{W}$ do not decay faster than the corresponding singular values $\sigma_{i}$ ), so the contribution of the noise is amplified and the solution is distorted [17]. The condition number of a matrix, defined as the ratio of its largest to its smallest singular value, $\sigma_{\max } / \sigma_{\min }$, gives a measure of the stability of the system to perturbations in the measurements. Because the amplification of the noise depends on the reciprocal of the singular values, as shown in (5), a large condition number means that the system is more unstable. The condition numbers of the forward model matrices used in this paper are larger than $10^{6}$, which means that the linear model is highly unstable and, hence, the use of the pseudoinverse of $\mathbf{S}$ to solve the problem is precluded.

The method for the inversion of the forward model has to be stabilized in order to obtain meaningful solutions. This can be accomplished by applying generalized Tikhonov regularization [18] to the MMSE inverse problem, which consist of modifying (3) such that

$$
\hat{\mathbf{r}}=\underset{r}{\arg \min }\left\{\|\mathbf{g}-\mathbf{S r}\|_{\mathbf{2}}^{\mathbf{2}}+\alpha^{2} J(\mathbf{r})\right\}
$$

The first term in (6) is referred to as the data discrepancy or fit-to-data function, and corresponds to the MMSE solution to the linearized problem. The second term is known as the regularization penalty or cost function, and is a stabilizing term that incorporates a priori knowledge about the signal to be recovered.

The type of generalized Tikhonov regularization that has been used in this paper corresponds to solving the following minimization problem [19]:

$$
\hat{\mathbf{r}}=\underset{r}{\arg \min }\left\{\|\mathbf{g}-\mathbf{S r}\|_{\mathbf{2}}^{\mathbf{2}}+\alpha^{2} \sum_{i=1}^{N}\left(\left|(\mathbf{L r})_{i}\right|^{2}+\beta\right)^{\frac{k}{2}}\right\} .
$$

This choice of the regularization cost function corresponds to the $l_{k}^{k}$ norm (which is defined as $\|\left.\mathbf{r}\right|_{k} ^{k} \triangleq \sum_{i=1}^{n}|\mathbf{r}|_{i}^{k}$ ) of a linear transformation of the vector of unknowns $\mathbf{r}$ using a matrix operator $\mathbf{L}$. The offset parameter $\beta$ is a small positive constant introduced to allow the differentiation of the regularization cost function with respect to $\mathbf{r}$ around zero for $k \leq 1$. The parameter $\alpha$ is known as the regularization parameter and controls the amount of regularization imposed on the solution.

The solution $\hat{\mathbf{r}}$ to the regularized inverse problem is found by evaluating the gradient of (7) with respect to $\mathbf{r}$, which results in

$$
\hat{\mathbf{r}}=\mathbf{S}^{\#} \mathbf{g}=\left[\left(\mathbf{S}^{\mathbf{T}} \mathbf{S}+\alpha^{2} \mathbf{L}^{\mathbf{T}} \mathbf{W}_{\beta}(\hat{\mathbf{r}}) \mathbf{L}\right)^{-1} \mathbf{S}^{\mathbf{T}}\right] \mathbf{g}
$$

where $\mathbf{S}^{\#}$ is the linear operator relating $\mathbf{g}$ and $\hat{\mathbf{r}}, \mathbf{W}_{\beta}(\hat{\mathbf{r}})=$ $(k / 2) \operatorname{diag}\left[\left(\left|(\mathbf{L} \hat{\mathbf{r}})_{i}\right|^{2}+\beta\right)^{1-(k / 2)}\right]$ and $\operatorname{diag}[\cdot]$ is a diagonal matrix whose $i$ th diagonal element is the expression inside the square brackets. Starting with an initial guess $\hat{\mathbf{r}}^{(0)}$, this nonlinear equation can be solved using the fixed point iteration

$$
\hat{\mathbf{r}}^{(n+1)}=\left[\left(\mathbf{S}^{\mathbf{T}} \mathbf{S}+\alpha^{2} \mathbf{L}^{\mathbf{T}} \mathbf{W}_{\beta}\left(\hat{\mathbf{r}}^{(n)}\right) \mathbf{L}\right)^{-1} \mathbf{S}^{\mathbf{T}}\right] \mathbf{g} .
$$

This process is stopped when the difference in the $l_{2}$ norm between successive iterations is small enough, that is, until $\left\|\hat{\mathbf{r}}^{(n+1)}-\hat{\mathbf{r}}^{(n)}\right\|_{2}^{2} /\left\|\hat{\mathbf{r}}^{(n+1)}\right\|_{2}^{2}<\delta$, where $\delta$ is the desired tolerance.

\section{Methods for the Selection of the Regularization Parameter}

The appropriate choice of values for the regularization parameter $\alpha$ is crucial to obtain good results. Even though for simulations one can estimate a range of optimal parameters by using visual inspection with a set of reference images, in practice the desired images are not available, so quantitative parameter selection methods need to be used. Several parameter selection techniques have been explored in the inverse problems literature, such as the discrepancy principle [20], the unbiased predictive risk estimator method (UPRE) [18], the generalized cross-validation method (GCV) [21], and the L-curve method [22]. Unlike the discrepancy principle and UPRE methods, GCV and the L-curve methods do not require knowledge of the statistics of the noise. Because of this, both the GCV and the L-curve methods were evaluated as regularization parameter selection techniques for this paper.

The L-curve method consists of plotting the $l_{2}$ norm of the penalty cost term versus the $l_{2}$ norm of the data discrepancy term, in a log-log scale. The resulting plot has typically an $\mathrm{L}$ shape, and the optimal value of $\alpha$ is chosen to be the one that corresponds to the corner of this curve, chosen as the point of maximum curvature. For Tikhonov regularization, even though the curve will have an $\mathrm{L}$ shape, it will be nonconvergent if the generalized Fourier coefficients of the forward operator do not satisfy the discrete Picard condition [23]. Also, when the residual is very small, the L-curve method fails to converge [24]. The applicability of this method has already been proposed for $l_{k}^{k}$ norm generalized Tikhonov regularization [25].

The GCV method is based on the minimization of the predictive error. Because the ideal data are not available, an estimate of the error is derived from the measurements as follows: for a fixed regularization parameter $\alpha$, the $i$-th equation is removed from the linear system $\mathbf{S r}=\mathbf{g}$. This corresponds to removing the $i$-th row of the forward model matrix $\mathbf{S}$ and the $i$-th entry of the measurements vector $\mathbf{g}$, which are denoted by $\mathbf{S}_{i}$ and $\mathbf{g}_{i}$, respectively. The solution to the reduced set of equations is denoted by $\hat{\mathbf{r}}^{(i)}$. The removed data point $\mathbf{g}_{i}$ can be estimated using $\hat{\mathrm{g}}_{i}=\mathbf{S}_{i} \hat{\mathbf{r}}^{(i)}$, with a corresponding estimation error. The predictive error is defined as the average of the estimation errors for all points in $\mathrm{g}$. The optimum value of $\alpha$ is the one that minimizes the predictive error because it can discriminate better the data from the noise in the measurements. The GCV estimator for the predictive error can be calculated as [18]

$$
\operatorname{GCV}(\alpha)=\frac{\|\mathbf{g}-\mathbf{S} \hat{\mathbf{r}}(\alpha)\|_{2}^{2}}{\left[\operatorname{trace}\left(\mathbf{I}-\mathbf{S}^{\mathbf{T}} \mathbf{S}^{\#}(\alpha) \mathbf{S}\right)\right]^{2}}
$$


with $\mathbf{S}^{\#}$ as given in (8). The optimum value of $\alpha$ is chosen to be the one that yields the minimum of the GCV curve. The GCV curve can have a very flat minimum, which makes it difficult to choose an appropriate parameter value. This method has been shown not to converge to the optimum parameter when the noise is correlated to the signal [26]. Also, the GCV method is computationally more expensive than the L-curve.

\section{Simulations AND RESUltS}

\section{A. Simulations Setup}

The simulated data were generated using Field II [27] using focused transducers in a medium with speed of sound equal to $1500 \mathrm{~m} / \mathrm{s}$. The simulated transducers had a center frequency of $6 \mathrm{MHz}$ (which corresponds to a wavelength $\lambda=250 \mu \mathrm{m}$ ) and a fixed focal length of $19 \mathrm{~mm}$. The ROIs were placed at the focus of the transducers for all the simulations. The sampling frequency was set to $60 \mathrm{MHz}$. The bandwidth and focal number of the transducers were not held constant for all simulations in order to analyze their impact on the quality of the reconstructions.

The spectrum of the transmit-receive impulse response of the transducer, $P(f)$, was modeled as a function of the frequency $f$ as

$$
P(f)=f \exp \left(-\frac{2.9\left(f-\kappa f_{0}\right)^{2}}{B W^{2}}\right)
$$

where $f_{0}$ is the center frequency of the transducer. The variable $\kappa$ in (11) is used to adjust the $-6 \mathrm{~dB}$ frequency band of the transducer. For this paper, $\kappa$ ranged between 0.9 and 1 .

The variations of the acoustic field were smoothed by apodizing the surface of the transducer. It is very common in signal processing to use Hann windows for reduction of sidelobes [1]. For this paper, the transducer was apodized using a modified Hann windowing function $A(r)$, defined as

$$
A(r)=\epsilon+(1-\epsilon) \cos \left(\frac{\pi r}{D}\right)
$$

where $D$ is the diameter of the transducer and $r \in[0,(D / 2)]$ is the radial distance from the center of the transducer. The variable $\epsilon$ in (12) is used to control the level of apodization. For this paper, $\epsilon$ was set to be 0.3 .

For speckle-based images using focused transducers, the correlation cell size was found to be on the same order as the spatial resolution at the focal region [28]. The expressions for the axial $(\Delta x)$ and lateral $(\Delta y)$ resolutions at the focus of the transducer are given by [29]

$$
\begin{aligned}
\Delta x & =\frac{c}{2 B W} \\
\Delta y & =1.028 \lambda f^{\#}
\end{aligned}
$$

where $c$ is the speed of sound and $f^{\#}$ is the focal number of the transducer, defined as the ratio of the focal length and the diameter of the transducer. The apodization applied to the surface of the transducer slightly increases the lateral size of the focal

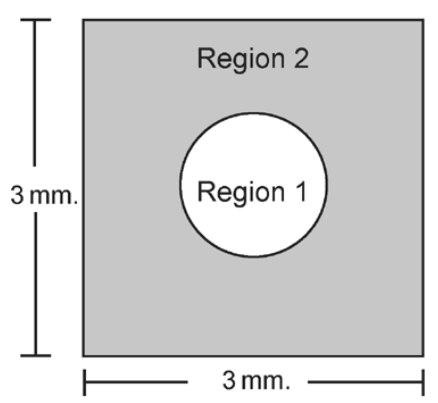

(a)

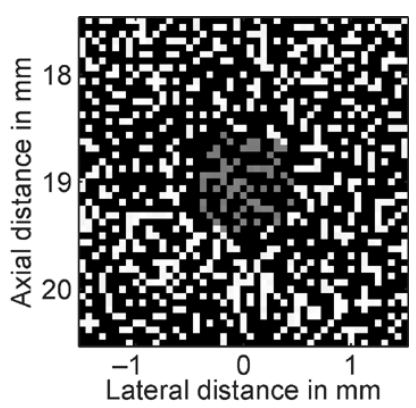

(b)
Fig. 2. (a) Schematic of the ROIs used for the simulations. (b) Sample reflectivity distribution used for visual evaluation of the reconstructions for the simulations.

region. Because the ROIs for this paper are 2-D, the size of the resolution cell can be approximated by $\Delta x \Delta y$.

The ROIs used for the simulations consist on 2-D, square regions of $3 \mathrm{~mm} \times 3 \mathrm{~mm}$, divided into two concentric subregions with the inner one having a diameter of $1 \mathrm{~mm}$, which corresponds to 4 wavelengths at the center frequency of the transducers used for the simulations. The mean reflectivity amplitudes are uniformly distributed in the intervals $[0.8,1]$ and $[0.08,0.1]$ for the outer and inner subregions, respectively. The subregions that constitute the ROIs are depicted in Fig. 2(a). The $\mathrm{B}$-mode images and regularization reconstructions displayed in the following subsections to visually evaluate the performance of the reconstructions correspond to the sample ROI shown in Fig. 2(b).

The positions of the scatterers in the ROIs were discretized using a grid of $\lambda / 4 \times \lambda / 4$ at the center frequency of the transducers, which results in images of $49 \times 49$ pixels. A total of 750 scatterers were randomly distributed in the ROIs, resulting in an average scatterer density of approximately 0.3125 scatterers per pixel. Because the highest $B W$ and the smallest $f^{\#}$ used in this paper are $100 \%$ and 1 , respectively, the smallest resolution cell used in the simulations corresponds roughly to a region of $0.5 \lambda$ $\times \lambda$. This means that a minimum average scatterer density of 2.5 scatterers per resolution cell is guaranteed for every simulation. This is above the limit of 2 scatterers per resolution cell determined by Tuthill et al. [30] for sparse scatterer density in speckle-based ROIs. The minimum value of 2.5 scatterers per resolution cell was also chosen to avoid using a large grid size which would increase the computation time of the algorithm.

\section{B. Evaluation of the Reconstructions}

The pulse-echo data $\mathrm{g}$ were generated by adding zero-mean Gaussian noise $\mathbf{w}$ to the data $\mathbf{y}$ predicted by the forward model. The signal-to-noise ratio (SNR) for this paper is defined as

$$
\mathrm{SNR}=10 \log \left(\frac{\|\mathbf{y}\|_{2}^{2}}{\|\mathbf{w}\|_{2}^{2}}\right)
$$

The noise realizations were scaled in order to change the SNR so that a fair comparison of the effect of several parameters could be performed.

Selected reconstructed images are displayed logarithmically with a dynamic range of $40 \mathrm{~dB}$ for a visual evaluation of the 
reconstructions. The numerical evaluation is performed based on the normalized mean square error (MSE), which is defined as

$$
\mathrm{MSE}=\frac{\|\mathbf{r}-\hat{\mathbf{r}}\|_{2}^{2}}{\|\mathbf{r}\|_{2}^{2}}
$$

where $\mathbf{r}$ and and $\hat{\mathbf{r}}$ are the ideal and reconstructed reflectivity distribution, respectively. The MSE was calculated using the data both in linear and logarithmic scale. The former is used because the reconstruction method is linear, and the latter because the images are shown in logarithmic scale. In order for the numerical results to have statistical meaning, 7 ROIs and 7 noise realizations were randomly generated, resulting in a total of 49 data sets used for each set of simulation settings. The average of the MSEs is used to quantify the performance of the reconstruction algorithm.

\section{Choice of the Parameters of the Regularization Method}

The regularization matrix $\mathbf{L}$ can be chosen as any stabilizing linear operator that includes a priori knowledge of the solution of the problem. In the case of speckle-based images, for which the reflectivity distribution has a random nature by definition, the optimum choice of $\mathbf{L}$ is not clear. The most natural way of regularizing an inverse problem is to remove the more unstable singular values as the level of noise in the data increases, which is know as TSVD regularization. A similar effect can be obtained in Tikhonov regularization by choosing the matrix $\mathbf{L}$ to be the identity matrix [31]. This choice of $\mathbf{L}$ has been used for the simulations in this paper.

The regularization cost norm $k$ was chosen by inspection of the effect of the norm on sample reconstructions. An example of the visual appearance of the reconstructions with values of $k$ of $0.5,1$, and 2 is shown in Fig. 3. The simulation corresponds to a reconstruction using a transducer with a $B W$ of $6 \mathrm{MHz}$ $B W / f_{0}=100 \%, f^{\#}$ of 1 and ROI at the focus, with a SNR of $20 \mathrm{~dB}$. This particular value of SNR was chosen because the difference in behavior is clearer when the amount of noise in the data is higher. The impact of the value of $k$ can be summarized as follows.

- From Fig. 3(c), for $k=2$ the solution looks oversmoothed. This case corresponds to the classical quadratic Tikhonov regularization, for which the minimization problem reduces to

$$
\hat{\mathbf{r}}=\underset{r}{\arg \min }\left\{\|\mathbf{g}-\mathbf{S r}\|_{2}^{2}+\alpha^{2}\|\mathbf{r}\|_{2}^{2}\right\} .
$$

The solution $\hat{\mathbf{r}}$ to (17) can be written in terms of the SVD of the forward model matrix $\mathbf{S}$ as

$$
\hat{\mathbf{r}}=\sum_{i=1}^{r} \frac{\sigma_{i}^{2}}{\sigma_{i}^{2}+\alpha^{2}} \frac{\mathbf{u}_{\mathbf{i}}^{\mathbf{T}} \mathbf{g}}{\sigma_{i}} \mathbf{v}_{\mathbf{i}}
$$

The last expression corresponds to a static filtering of the most unstable singular values. Because these singular values are the ones that carry the high-frequency information of the image, filtering them results in blurred images.

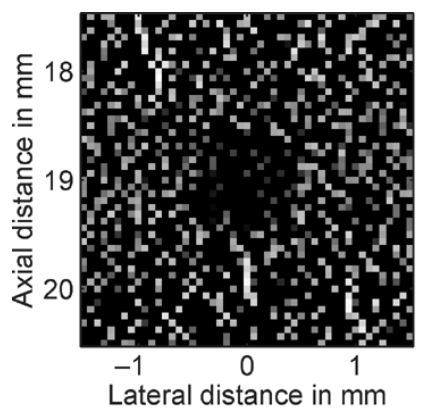

(a)

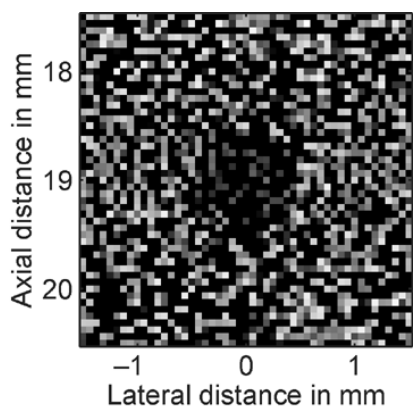

(b)

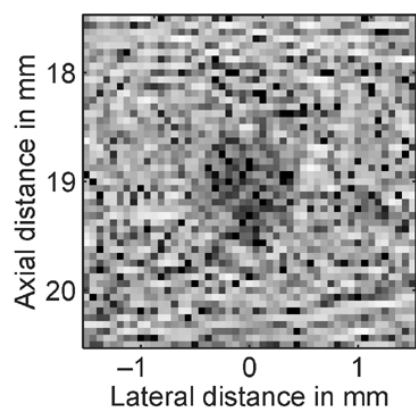

(c)

Fig. 3. Effect of different choices of regularization cost norm $k$ in the reconstruction of the reflectivity distribution. The optimum reconstruction was chosen using the L-curve criterion. Reconstructions using (a) $k=0.5$ (MSE(linear) $=99 \%, \operatorname{MSE}(\log )=76 \%$ ) (b) $k=1(\operatorname{MSE}($ linear $)=54 \%, \operatorname{MSE}(\log )=55 \%)($ c) $k=2$ $(\operatorname{MSE}($ linear $)=50 \%, \operatorname{MSE}(\log )=102 \%)$.

- From Fig. 3(a), for $k=0.5$ the solution looks sparse; i.e., there is no smoothing effect on the reconstructed image at the cost of losing many features from the original image. This effect can be explained better starting from (7). The regularization cost function when $\mathbf{L}$ is chosen to be the identity matrix and $\beta=0$ simplifies to $\|\mathbf{r}\|_{k}^{k}$, that is, the $l_{k}^{k}$ norm of the data to be recovered. Because the solution to the minimization equation is no longer linear, the singular value filtering process becomes image dependent, extracting information even from the unstable singular values, resulting in solutions that are not oversmoothed. Smaller values of $k$ enforce a larger emphasis of the cost function. Because the minimization of the cost function will be accomplished for smaller values of $\|\mathbf{r}\|_{k}^{k}$ (sparser solutions), the sparsity in the reconstructions will be larger when $k$ is smaller.

- From Fig. 3(b), for $k=1$ an balance between the aforementioned behaviors is achieved. The solution is somewhat sparse as in the case of $k=0.5$ and suffers from a slight blurring which however adds visual cohesion to the image. The average normalized MSEs calculated using the data in linear scale are $99 \%, 54 \%$, and $50 \%$ for values of $k$ of 0.5 , 1 , and 2 , respectively. However, the average normalized MSE calculated using the data in logarithmic scale in the reconstruction with $k=1(55 \%)$ is smaller than the one for the case of $k=0.5(76 \%)$ and $k=2(102 \%)$. Hence, a value of $k=1$ allows for a significantly better performance in terms of the MSE in logarithmic scale only at a slight cost of accuracy in linear scale. This value of $k$ will be used for the rest of the simulations in this paper. 
Linear

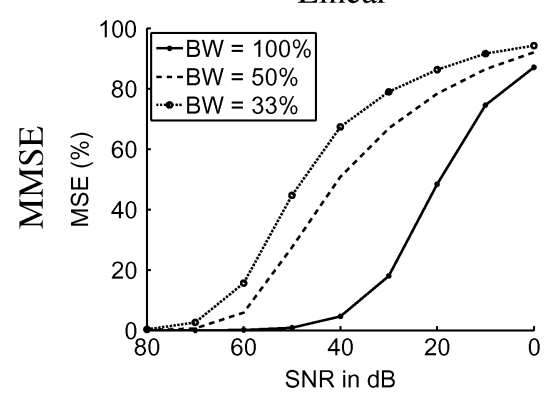

(a)
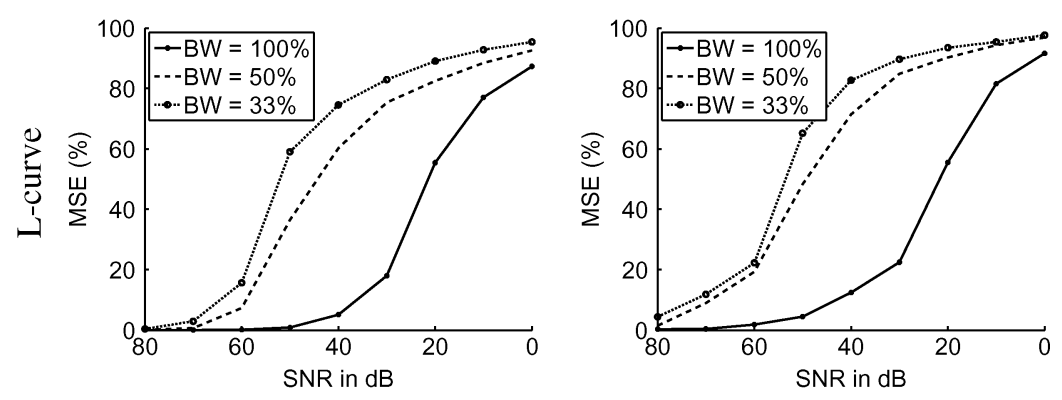

(b)
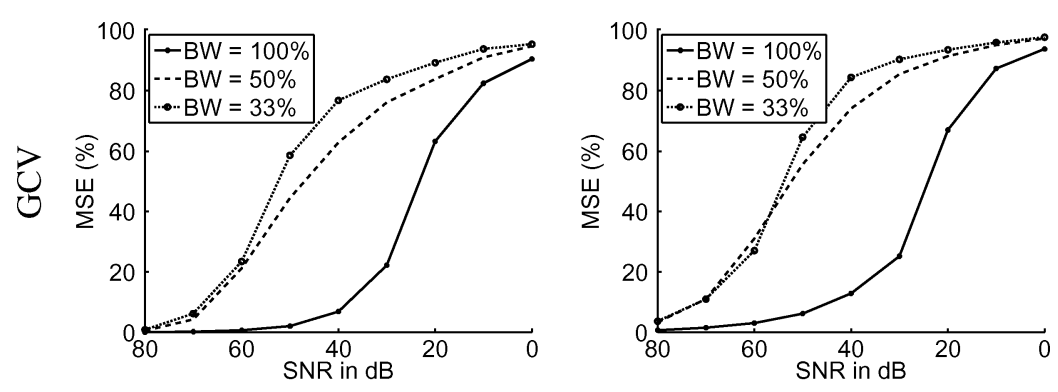

(c)

Fig. 4. Effect of the transmit-receive bandwidth of the transducer in the reconstructions. Average normalized MSE curves corresponding to the regularization reconstructions using relative transmit-receive bandwidths of $100 \%, 50 \%$, and $33 \%$, and a focal number of 1 . First column: MSE calculated using the data in linear scale. Second column: MSE calculated using the data in logarithmic scale. The optimum reconstructions were selected using (a) the MMSE criterion, (b) the L-curve method, and (c) the GCV method.

The value of $\beta$ has to be chosen large enough to avoid instability in the reconstruction process, but small enough to avoid biasing the solutions. As $k$ increases, the sensitivity to variations in $\beta$ decreases. An empirical value of $\beta=10^{-7}$ was selected for this paper.

The interval of analysis for the regularization parameter $\alpha$ was determined through simulations. For $\alpha<10^{-4}$ the regularization effect is too weak and the distortion on the output due to noise amplification is excessive even for a SNR of $100 \mathrm{~dB}$. For $\alpha>10$, the regularization constraint term is too large and the MSE in linear scale reaches an asymptotic limit of $100 \%$. The values of the parameter $\alpha$ were discretized using a uniform exponential grid with step size equal to 0.25 , that is, $\alpha=$ $\left\{10^{-4}, 10^{-3.75}, 10^{-3.5}, \ldots, 10^{0.75}, 10^{1}\right\}$.

\section{Effect of the BW of the Transducer}

The effect of the $B W$ of the transducer is analyzed by reconstructing images from simulated data corresponding to scanning the ROIs with focused transducers with an $f^{\#}$ of 1 and relative $B W \mathrm{~s}\left(B W_{R}\right)$ of $100 \%, 50 \%$, and $33 \%$, respectively, where
$B W_{R}=B W / f_{0}$. When compared to the case of $B W_{R}=$ $100 \%$, using $B W_{R}$ s of $50 \%$ and $33 \%$ corresponds to increasing the resolution cell size by a factor of 2 and 3, respectively. Fig. 4 shows the plots of the average MSE as a function of the SNR for the regularization reconstructions. Fig. 5 shows sample regularization reconstructed images selected using the L-curve criterion for each simulated transducer for selected values of SNR of 50, 30, and $10 \mathrm{~dB}$. Fig. 6 shows the ultrasonic pulses used in the simulations in both time and frequency domain.

Several observations can be made from the results of these simulations. First, from Fig. 4 it is clear that the error in the reconstruction increases monotonically with the level of noise in the measurements, as expected. This can also be visually verified by analyzing the quality of the images in Fig. 5; the images corresponding to higher SNRs represent better the underlying reflectivity distribution.

Second, it can be seen that the quality of the reconstruction degrades as the bandwidth decreases; this trend is more noticeable in the plots of the average MSE calculated from the data in linear scale, where the separation of the error curves corresponding to $B W_{R}$ of $50 \%$ and $33 \%$ is more pronounced. As a 


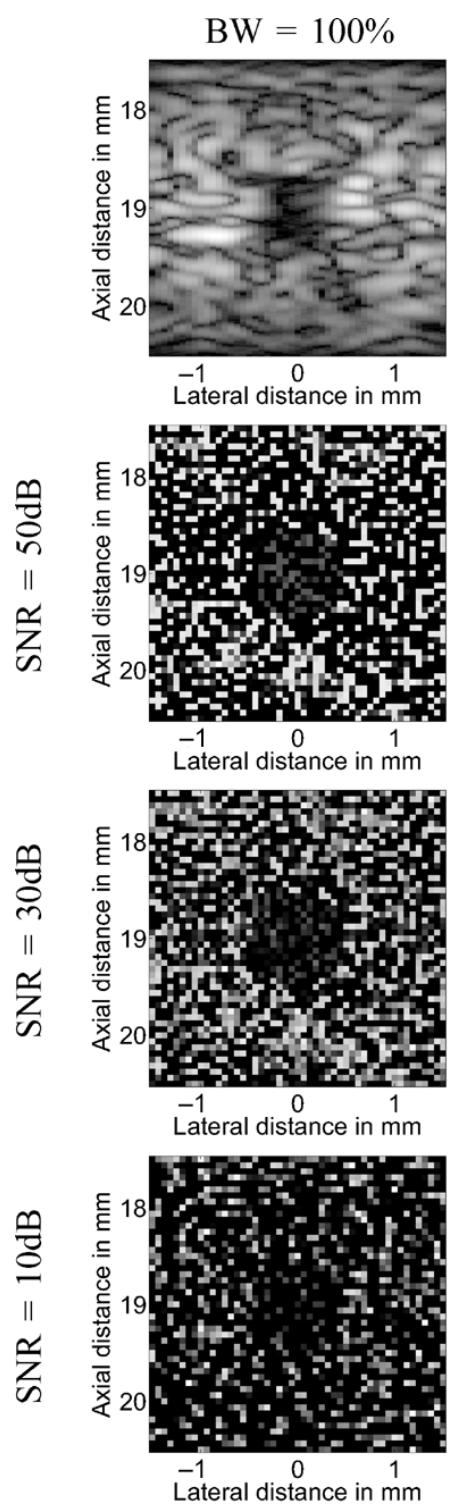

(a)
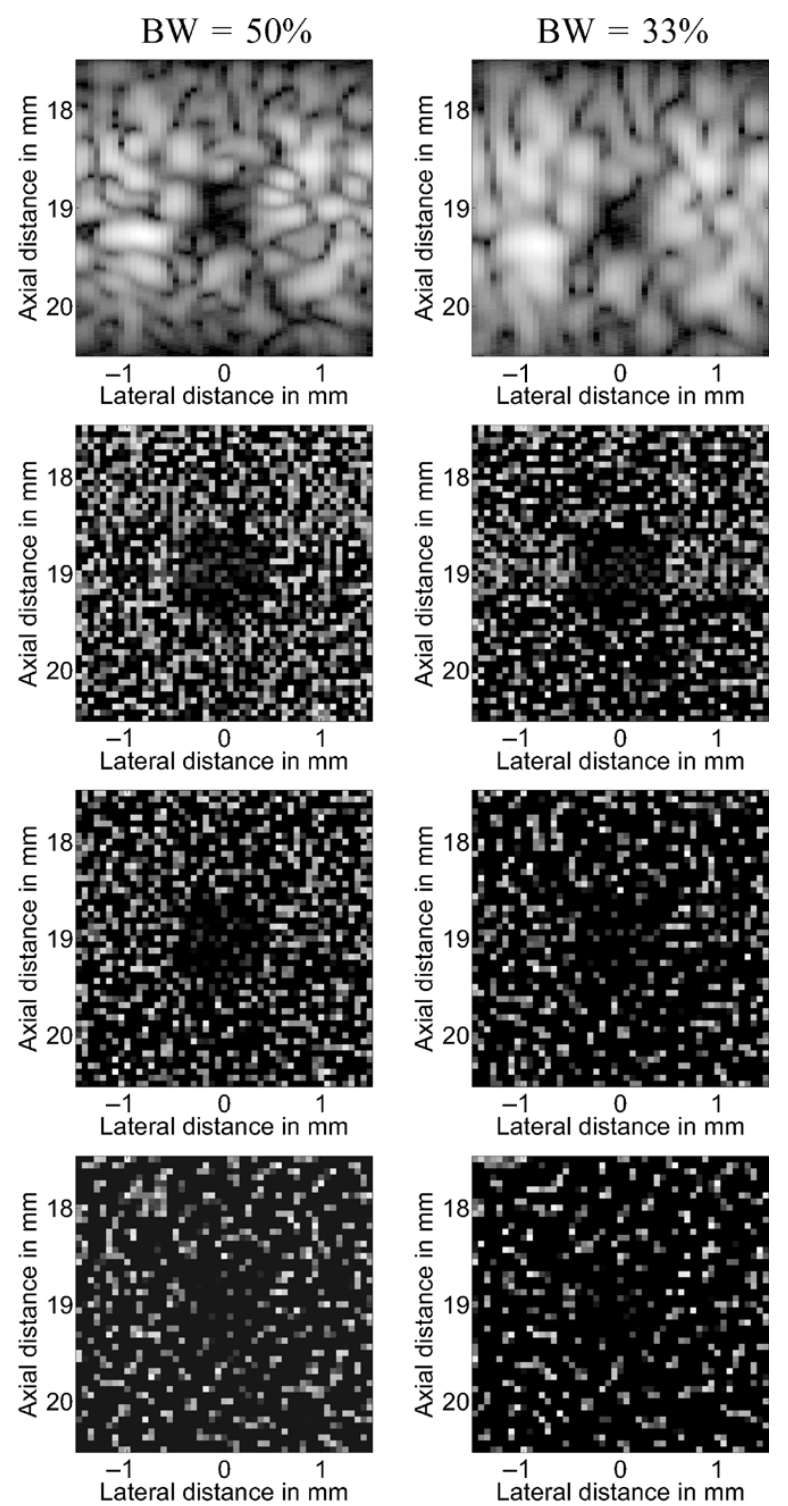

(b)

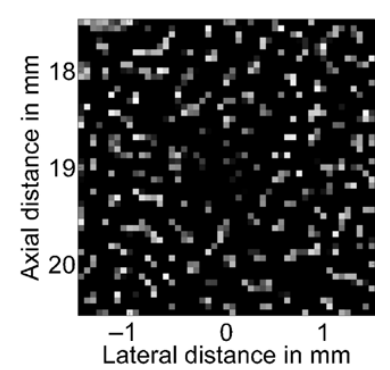

(c)

Fig. 5. Effect of the transmit-receive bandwidth of the transducer in the reconstructions. Reconstructed images formed using a focal number of 1 and relative transmit-receive bandwidths of (a) 100\%, (b) 50\%, and (c) 33\%. First row: B-mode images at a SNR of $30 \mathrm{~dB}$. Second to fourth row: regularization reconstructions at SNRs of 50, 30, and $10 \mathrm{~dB}$, respectively.

numerical example of the effect of the $B W$ on the reconstruction error, for a SNR of $30 \mathrm{~dB}$ the average normalized MMSE using the data in logarithmic scale is $22.5 \%$ for a $B W_{R}$ of $100 \%$ but it increases to $86.5 \%$ when the $B W_{R}$ is $33 \%$. For the data in linear scale, the corresponding average normalized MMSEs are $18 \%$ and $79 \%$. This behavior is expected because the forward model matrix is a spatially variant convolution kernel, which is more stable if its frequency content is larger. According to (13), reducing the $B W$ of the transducer will increase the axial duration of the impulse response and, hence, reduce the spatial frequency content in the axial direction. This reduces the stability of the forward model and, hence, increases the error of the inversion algorithm. The results show that the algorithm is very sensitive to the frequency content of the ultrasonic pulse.

Third, it can be seen from the reconstructions for SNRs of 50 and $30 \mathrm{~dB}$ that the low amplitude pixels are discarded before the high amplitude ones. This is consistent with the sparsity con- straint enforced by the regularization cost function, as discussed in Section III-C.

\section{E. Effect of the $f^{\#}$ of the Transducer}

Just like the axial resolution of the system is dependent on the $B W$ of the transducer, the $-6 \mathrm{~dB}$ lateral resolution at the focal region depends on the $f^{\#}$ of the transducer as given in (14). Hence, increasing the $f^{\#}$ should also have an effect on the reconstruction algorithm. To evaluate this effect, simulations were performed with focused transducers with a $B W_{R}$ of $100 \%$ and $f^{\#}$ of 1,2 , and 3, respectively. When compared to the case of $f^{\#}=1$, using $f^{\#}$ s of 2 and 3 corresponds to increasing the resolution cell size by a factor of 2 and 3, respectively, which are equal to the factors obtained when changing the $B W_{R}$ in the previous set of simulations. It is worth noticing that for three-dimensional (3-D) ROIs, the size of the ROI depends quadratically on the $f^{\#}$ (and not linearly as in the 2-D case) and, hence, the 

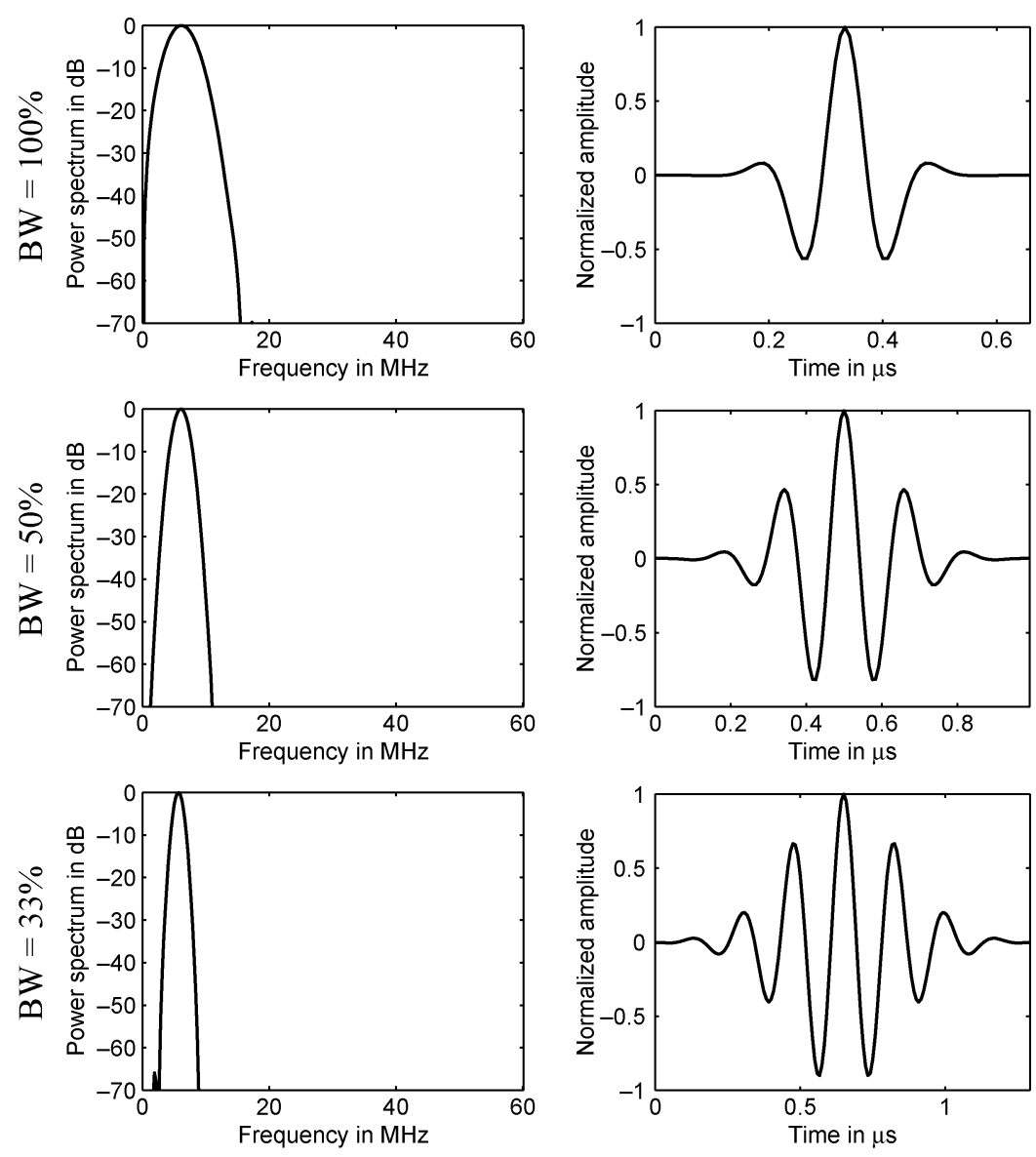

(a)

(b)

Fig. 6. (a) Time domain waveforms and (b) power spectrum of the pulses used in the simulations. The pulses correspond to relative transmit-receive BWs of $100 \%$ (first row), (b) $50 \%$ (second row), and (c) $33 \%$ (third row).

scaling factors would be larger. The average MSE versus SNR curves and sample reconstructed images are shown in Figs. 7 and 8 , respectively.

Some important observations can be derived from the results of these simulations. First, it can be noticed from visual inspection of the first row of Fig. 8 that the B-mode images corresponding to $f^{\#}$ s of 2 and 3 do not show a distinction of the subregions in the ROI. This is not the case for the images reconstructed using regularization for high values of SNR. This is a clear example of the possibility of extracting more information from the pulse-echo data than the one that can be obtained using B-mode imaging.

Second, it can be seen that the effect on the performance of the increase in $f^{\#}$ is not nearly as critical as the one obtained in the simulations for the reduction of the $B W$. As a numerical example, with a SNR of $30 \mathrm{~dB}$ the average normalized MMSE using the data in logarithmic scale increases from $22.5 \%$ to $37.8 \%$ when the $f^{\#}$ increases from 1 to 3 ; for the data in linear scale, the corresponding average normalized MMSEs are $18 \%$ and $43.9 \%$. This is to be compared against the average MSEs of $86.5 \%$ in logarithmic scale and $79 \%$ in linear scale for the case of changing the $B W_{R}$ to $33 \%$. Also, it can be seen that the separation between the curves in both linear and logarithmic scale is very similar. The difference in behavior can be explained because more destructive interference is expected when using a pulse with a smaller $B W$ due to the increase in coherence, as it can be seen from Fig. 6. This corresponds to an increase in the dimension of the nullspace of the forward model matrix $\mathbf{S}$. In contrast, the increase of the $f^{\#}$ does not affect the coherence of the acoustic pulse; hence, the destructive interference of the received echoes does not increase and the stability of the algorithm is not severely degraded. The error curve for $f^{\#}$ of 1 is separated from the curves for $f^{\#} \mathrm{~s}$ of 2 and 3 ; these last two curves, however, are completely overlapping.

\section{F. Comparison of the Performance of the L-Curve and GCV Methods}

As a final remark on the selection of the regularization parameter, it can be seen from the error curves for all the simulations that there is not a significant difference in the performance of the L-curve and GCV methods. Even though the L-curve method seems to track better the MMSE curves, both methods select regularization parameters that yield reconstructions with comparable levels of error.

\section{CONCLUSION}

The performance of B-mode imaging is mainly limited by the size of the resolution cell of the imaging system. For monostatic imaging with a focused transducer, the spatial resolution depends on the wavelength, the $f^{\#}$, and the $B W$. The goal of 
Linear

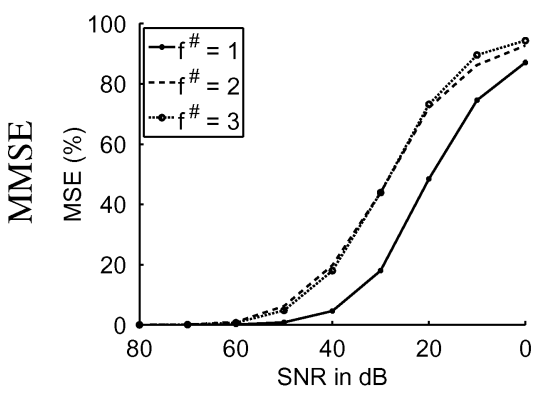

$\log$

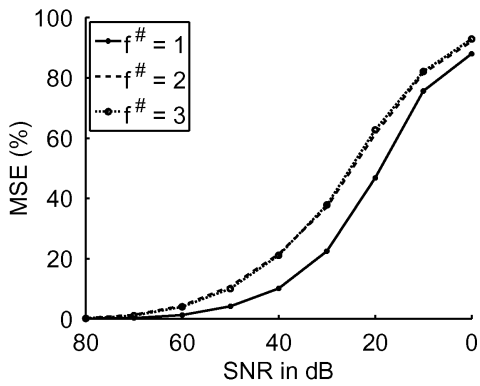

(a)
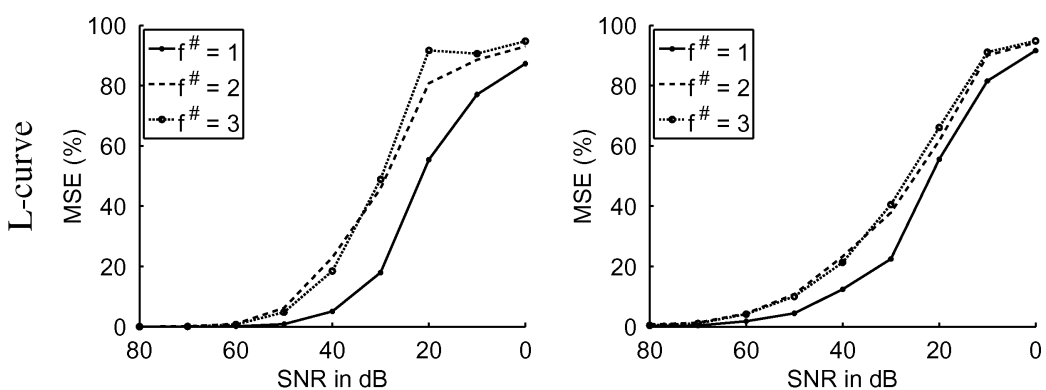

(b)
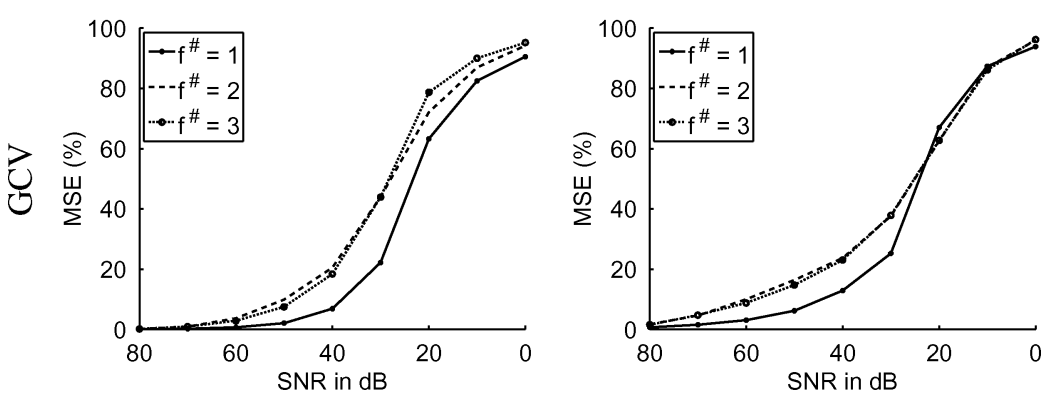

(c)

Fig. 7. Effect of the focal number of the transducer in the reconstructions. Average normalized MSE curves corresponding to the regularization reconstructions using $f^{\#}$ of 1,2, and 3, and a relative transmit-receive bandwidth of $100 \%$. First column: MSE calculated using the data in linear scale. Second column: MSE calculated using the data in logarithmic scale. The optimum reconstructions were selected using (a) the MMSE criterion, (b) the L-curve method, and (c) the GCV method.

this paper was to analyze if regularization approaches are a feasible alternative to B-mode imaging when the $\mathrm{ROI}$ is at the focus of the transducer.

It was found from the simulations that more information can be extracted from the pulse-echo data using a regularization approach compared to conventional techniques when the SNR is high. However, as the noise in the measurements increases, the MSE of the reconstructions also increases and the quality of the reconstruction degrades because of measurement noise amplification and loss of features of the image. This mechanism of image distortion is not present in conventional imaging techniques.

The $B W$ and $f^{\#}$ of the imaging system also have an impact on the performance of regularization techniques by determining the lower threshold for the SNR for which these approaches yield an image of better quality. This is a direct result of a lower spatial frequency content of the forward model which results in a more unstable inverse problem. A larger $B W$ results in a better performance as assessed by the MSE for fixed SNR. The effect of the $f^{\#}$ in the performance of the reconstruction was found to be less severe.
The optimum reconstructions for the simulations occurred for a $B W_{R}$ of $100 \%$ and an $f^{\#}$ of 1 . For these settings and values of SNR down to $40 \mathrm{~dB}$, the average MMSE is below $10 \%$ in both linear and logarithmic scales and good reconstructions are obtained. For SNRs below $20 \mathrm{~dB}$, the average MMSE is above $70 \%$ and the reconstructions appear severely distorted. A SNR of $30 \mathrm{~dB}$ has been chosen as a lower threshold because it is contained on the lower portion of the MMSE curve that serves as a transition for the aforementioned behaviors.

It should be noted that whereas B-mode imaging only involves envelope extraction from the measured waveforms, which is a very inexpensive operation, the proposed inversion method involves solving large matrix systems of equations and, hence, is computationally costly. This implies that, unlike conventional B-mode imaging, this technique in its current implementation is not suitable for real-time processing, but can be used as a complementary off-line tool. Our primary goal in this paper was to assess the potential of the inverse technique for improving the image formation. A detailed analysis of computational considerations and tradeoffs is the subject of a future study. 


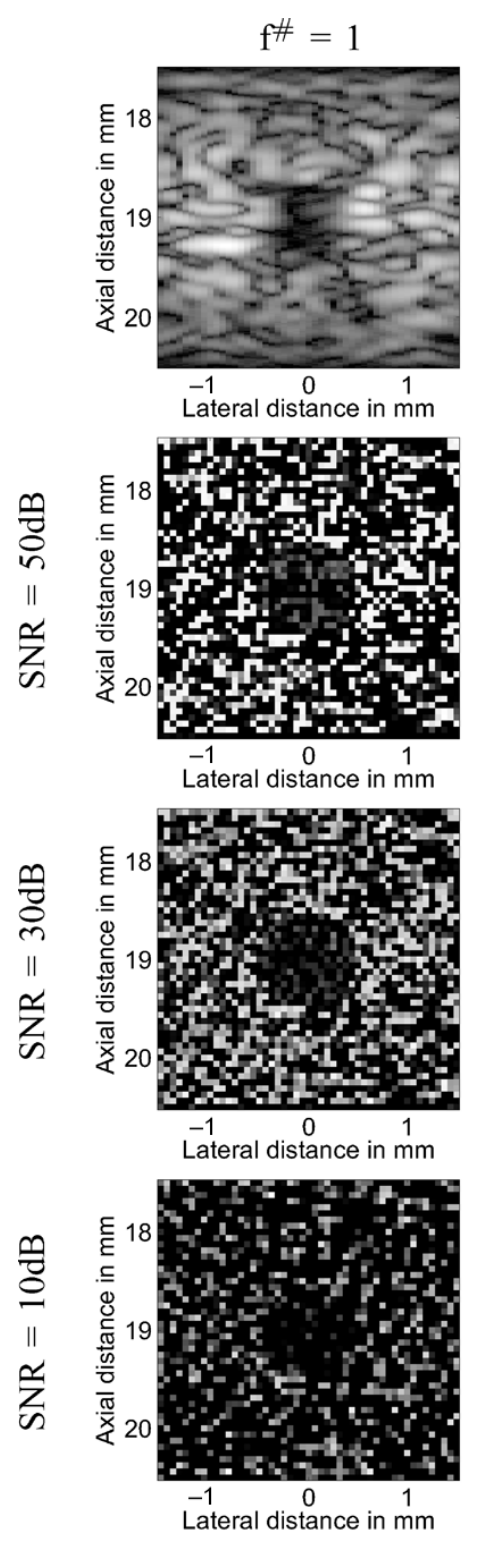

(a)
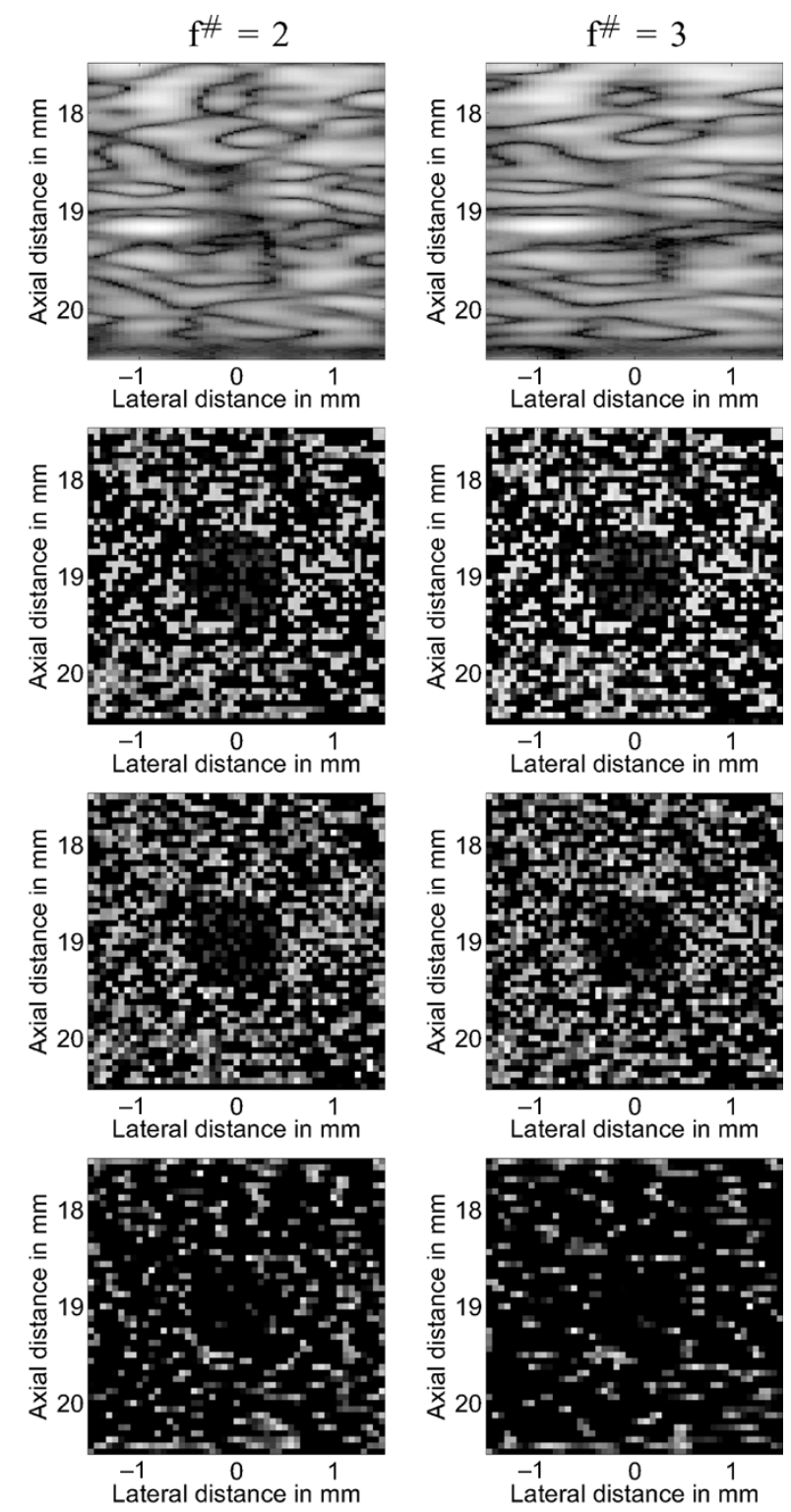

(b)

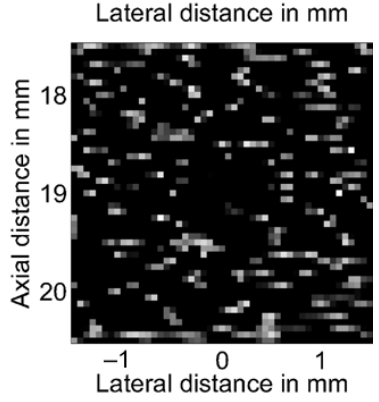

(c)

Fig. 8. Effect of the focal number of the transducer in the reconstructions. Reconstructed images formed using a relative transmit-receive bandwidth of $100 \%$ and a focal number of (a) 1, (b) 2, and (c) 3. First row: B-mode images at a SNR of $30 \mathrm{~dB}$. Second to fourth row: regularization reconstructions at SNRs of 50, 30, and $10 \mathrm{~dB}$, respectively.

This method can in principle be extended to deal with 3-D distributions in a straight forward manner by replacing the 2-D spatially variant impulse response matrix of (2) by the corresponding 3-D one. However, the computational expense and memory requirement of the algorithm would increase. This issue would have to be studied to analyze the feasibility of the reconstruction of 3-D volumes with this technique.

Both conventional B-mode imaging and the regularization reconstructions provide information about the ROI. The speckle in B-mode images has been shown to provide useful information for clinical analysis [32], [33]. For the simulations in this paper, the regularization reconstructions provide information about the localization of scatterers and, for the case of the $f^{\#}$ simulations, a better distinction among the subregions of the ROI. A combination of both imaging techniques may provide a better tool for the evaluation of pulse-echo ultrasonic data.

\section{ACKNOWLEDGMENT}

The authors gratefully acknowledge the comments and suggestions of the anonymous reviewers, which aided the improvement of the article.

\section{REFERENCES}

[1] G. S. Kino, Acoustic Waves: Devices, Imaging, and Analog Signal Processing. Englewood Cliffs, NJ: Prentice-Hall, 1987.

[2] A. Macovski, "Ultrasonic imaging using arrays," Proc. IEEE, vol. 67, no. 4, pp. 484-495, Apr. 1979.

[3] C. Frazier and W. D. O'Brien, Jr., "Synthetic aperture techniques with a virtual source element," IEEE Trans. Ultrason., Ferroelect., Freq. Control, vol. 45, no. 1, pp. 196-207, Jan, 1998.

[4] C. B. Burckhard, "Speckle in ultrasound B-mode scans," IEEE Trans. Sonics Ultrason., vol. 25, no. 1, pp. 1-6, Jan. 1978.

[5] F. Lingvall, T. Olofsson, and T. Stepinski, "Synthetic aperture imaging using sources with finite aperture: deconvolution of the spatial impulse response," J. Acoust. Soc. Am., vol. 114, no. 1, pp. 225-234, July 2003. 
[6] H. Desoky, A. B. M. Youssef, and Y. M. Kadah, "Reconstruction using optimal spatially variant kernel for B-mode ultrasound imaging," Proc. SPIE (Medical Imaging 2003: Ultrasonic Imaging and Signal Processing), pp. 147-153, 2003.

[7] Y. Wang and W. Chew, "Reconstruction of two-dimensional refractive index distribution using the Born iterative and distorted Born iterative method," Acoust. Imag., vol. 18, pp. 105-114, 1991.

[8] X. Zhang and S. Broschat, "A comparison of material classification techniques for ultrasound inverse imaging," J. Acoust. Soc. Am., vol. 111, no. 1, pp. 457-467, January 2002.

[9] C. Liu, Y. Wang, and P. A. Heng, "A comparison of truncated total least squares with Tikhonov regularization in imaging by ultrasound inverse scattering," Phys. Med. Biol., vol. 48, no. 15, pp. 2437-2451, Aug. 2003.

[10] Z. Mu, R. Plemmons, D. Herrington, and P. Santago, "Estimation of complex ultrasonic medium responses by deconvolution," in Proc. IEEE Int. Symp. Biomedical Imaging, Jul 2002, pp. 1047-1050.

[11] O. Husby, T. Lie, T. Lang, J. Hokland, and H. Rue, "Bayesian 2-D deconvolution: a model for diffuse ultrasound scattering," IEEE Trans. Ultrason., Ferroelect., Freq. Control, vol. 48, no. 1, pp. 121-130, Jan. 2001.

[12] J. Shen and E. S. Ebbini, "A new coded-excitation ultrasound imaging system-part I: basic principles," IEEE Trans. Ultrason., Ferroelect., Freq. Control, vol. 43, no. 1, pp. 131-140, Jan. 1996.

[13] A. Jarrot, H. Liebgott, P. Delachartre, and M. Robini, "Edge-preserving image reconstruction for Young's modulus recovery from ultrasonic data," in Proc. IEEE Ultrason. Symp., 2003, pp. 1919-1922.

[14] W. C. Karl and M. Cetin, "Edge preserving image reconstruction for coherent imaging applications," in Proc. IEEE Int. Conf. Image Processing, Sep. 2002, pp. 481-484.

[15] R. Lavarello, F. Kamalabadi, and W. D. O’Brien, Jr., "A study of pulseecho image formation using nonquadratic regularization with specklebased images," Acoust. Imag., vol. 28, 2006, to be published.

[16] D. S. Watkins, Fundamentals of Matrix Computations. New York: Wiley, 2002.

[17] P. C. Hansen, Rank-Deficient and Discrete Ill-Posed Problems: Numerical Aspects of Linear Inversion. Philadelphia, PA: SIAM, 1988.

[18] C. R. Vogel, Computational Methods for Inverse Problems. Philadelphia, PA: SIAM, 2002.

[19] W. C. Karl and M. Celin, "Feature-enhanced synthetic aperture radar image formation based on nonquadratic regularization," IEEE Trans. Image Process., vol. 10, no. 4, pp. 623-631, Apr. 2001.

[20] V. A. Morozov, "On the solution of functional equations by the method of regularization," Soviet Mathematics—Doklady, vol. 7, pp. 414-417, 1966.
[21] G. H. Golub, M. Heath, and G. Wahba, "Generalized cross-validation as a method for choosing a good ridge parameter," Technometrics, vol. 21, pp. 215-223, 1979.

[22] C. L. Lawson and R. J. Hanson, Solving Least Squares Problems. Philadelphia, PA: Prentice-Hall, 1974.

[23] C. R. Vogel, "Non-convergence of the L-curve regularization parameter selection method," Inverse Prob., vol. 12, no. 4, pp. 535-547, August 1996.

[24] M. E. Kilmer and D. P. O'Leary, "Choosing regularization parameters in iterative methods for ill-posed problems," SIAM J. Matrix Anal. Applicat., vol. 22, no. 4, pp. 1204-1221, Apr. 2001.

[25] S. Oraintara, W. C. Karl, D. A. Castanon, and T. Q. Nguyen, "A method for choosing the regularization parameter in generalized Tikhonov regularized linear inverse problems," in Proc. 2000 Int. Conf. Image Processing, Sep. 2000, pp. 93-96.

[26] P. C. Hansen, "Analysis of discrete ill-posed problems by means of the L-curve," SIAM Rev., vol. 34, no. 4, pp. 561-580, Dec. 1992.

[27] J. A. Jensen and N. B. Svendsen, "Calculation of pressure fields from arbitrarily shaped, apodized, and excited ultrasound transducers," IEEE Trans. Ultrason., Ferroelect., Freq. Control, vol. 39, no. 2, pp. 262-267, Mar. 1992.

[28] R. F. Wagner, S. W. Smith, J. M. Sandric, and H. Lopez, "Statistics of speckle in ultrasound B-scans," IEEE Trans. Sonics Ultrason., vol. 30, no. 3, pp. 153-163, May 1983.

[29] K. Raum and W. D. O'Brien, Jr., "Pulse-echo field distribution measurement technique for high-frequency ultrasound sources," IEEE Trans. Ultrason., Ferroelect., Freq. Control, vol. 44, no. 4, pp. 810-815, Jul. 1997.

[30] T. A. Tuthill, R. H. Sperry, and K. I. Parker, "Deviations from Rayleigh statistics in ultrasonic speckle," Ultrason. Imag., vol. 10, no. 2, pp. 81-89, Apr. 1988.

[31] H. W. Engl, M. Hanke, and A. Neubauer, Regularization of Inverse Problems. Boston, MA: Kluwer Academic, 1996.

[32] F. Sommer, L. Joynt, B. Carroll, and A. Macovski, "Ultrasonic characterization of abdominal tissues via digital analysis of backscattered waveforms," Radiology, vol. 141, no. 3, pp. 811-817, Dec. 1981.

[33] P. Shankar, J. Reid, H. Ortega, C. Piccoli, and B. Goldberg, "Use of non-Rayleigh statistics for the identification of tumors in ultrasonic B-scans of the breast," IEEE Trans. Med. Imag., vol. 12, no. 4, pp. 687-692, Dec. 1993 